ISSN 1991- 8690

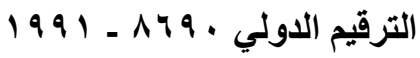

website : http:// jsci.utq.edu.iq

Email: utjsci@utq.edu.iq

\title{
تحسين كفاءة كاشف الأثعة فوق البنفسجية بزيادة الحساسية البصرية
}

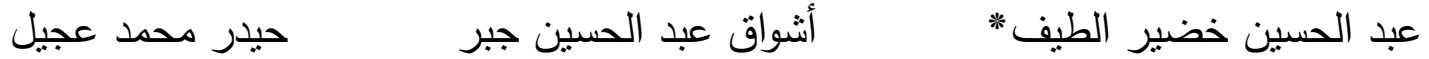

الخلاصة

تم في هذا البحث تحضير أغشية اوكسيد القصدير (SnO2) الرقيقة بطريقة الرش الكيميائي الحراري من رش محلول مكون من مادة كلوريدات

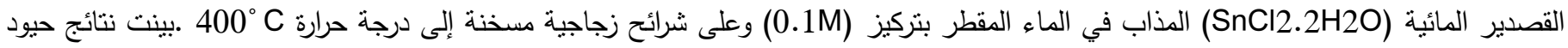

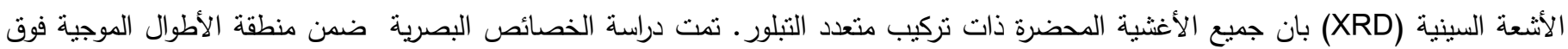

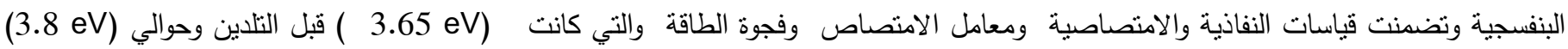
بعد التلدين. كذللك تم قياس خصائص الاستجابة الطيفية للكواثف المصنعة في مدى الأشعة فوق البنفسجية وكانت الاستجابة الطيفية لها بحدود

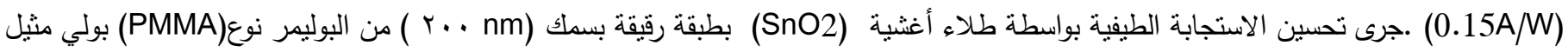
ميثا اكريلايت ذي الامتصاصية العالية لتلك للأشعة، حيث وصلت الاستجابة الطيفية إلى (1.62 A/W) عند الطول ألموجي (365nm) وفولتية انحياز

\section{$\underline{\text { Abstract }}$}

In this work, $\left(\mathrm{SnO}_{2}\right)$ thin films were prepared by thermal chemical spray pyrolysis technique using mixture of Tin chloride hydrate $\left(\left(\mathrm{SnCl}_{2} \cdot 2 \mathrm{H}_{2} \mathrm{O}\right)\right)$ with molar concentration of $(0.1 \mathrm{M})$ at glass substrate temperature of $400{ }^{\circ} \mathrm{C}$. $\mathrm{X}$-ray diffraction analysis indicated that all the prepared films have polycrystalline structure. Optical properties of the films were determined by studying the ultraviolet spectrum which include transmittance, absorptance, absorption coefficient and energy gap which have a value of $(3.65 \mathrm{eV})$ before annealing and about $(3.8 \mathrm{eV})$ after annealing.The spectral responsivity of the uncoated detector in UV range was $(0.15 \mathrm{~A} / \mathrm{W})$. The photo response enhancement of the photoconductive detector is improved by coating $\left(\mathrm{SnO}_{2}\right)$ films with 200nm (PMMA) polymer . The results show that the responsivity is increased more than responsivity of the uncoated $\left(\mathrm{SnO}_{2}\right)$ and it reaches $(1.62 \mathrm{~A} / \mathrm{W})$ at $(385 \mathrm{~nm})$ and at (10V) bias voltage.

ومميزاتها لتؤدي الغرض الذي استعملت من اجله وان اختبار الطريقة

المقدمة

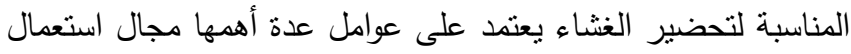

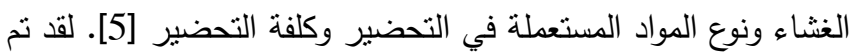

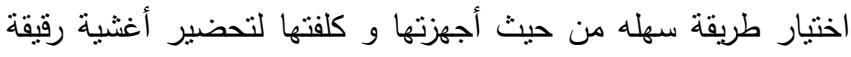
من مادة (SnO2) وهي طريقة الرش الكيميائي الحراري حيث تمناز هذه الطريقة بأنه يمكن بواسطتها تحضير أغشية مواد ذات درجات انصهار عالية وذات تجانس جيد وبمساحات كبيرة. يمكن استخدام هذه الطريقة في الظروف الجوية الاعتيادية .إن من أهم الفوائد لاستخدام

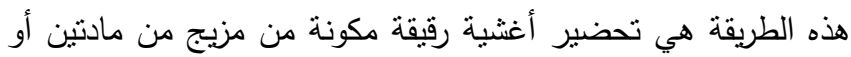

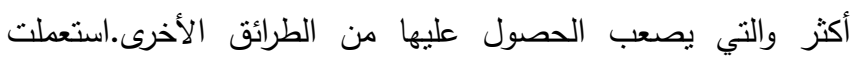

$$
\begin{aligned}
& \text { تعتبر مادة (SnO2) من اكاسيد التوصيل الثفافة ذات فجوة }
\end{aligned}
$$

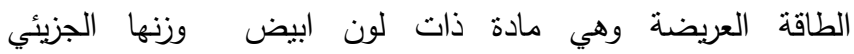

$$
\begin{aligned}
& \text { 1630C وكثافتها (6.95g/cm3) ودرجة انصهارها (150.7g/mol) } \\
& \text { ودرجــة غليانها [11 C C C (1900-1800) • استعملت أغشية } \\
& \text { (SnO2) الرقيقة في كثير من الأجهزة الكهروبصرية وفي الخلايا } \\
& \text { الثمسية إضافة إلى أجهزة التحس والكثف عن التهن الغازات وتطبيقات } \\
& \text { صناعية أخرى [2-4] - ونتيجة للنطور العلمي فقد تطورت طرق إلى } \\
& \text { تحضير الأغثية الرقيقة وأصبحت على درجة عالية من الدقة في تحديد }
\end{aligned}
$$

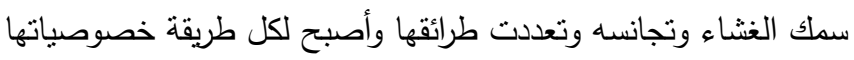


طاقة الفوتون الساقط (hv) وعلى خصائص شبه الموصل من حيث نوع الانتقالات الإككترونية التي تحدث بين حزم طاقئ طاقاته وفجوة الطاقة

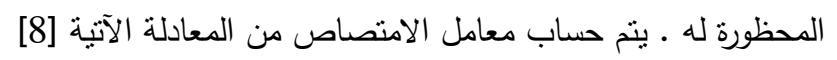

$$
\alpha=2.303 \frac{A}{t}
$$

يتم حساب قيم ( $)^{\alpha}$ لكل نسبة تتويب وذلك من خلال أيجاد الطاقة للطول الموجي الأحادي للصوديوم (589.3 nm) باستخدام معادلة

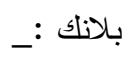

$\mathrm{E}=\mathrm{hc} / \lambda$

r- حساب فجوة الطاقة المحظورة للانتقالات الالكترونية المباشرة :

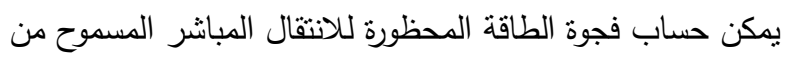

المعادلة الآتية [8ن

$$
(\alpha h v)=A(h v-E g) r
$$

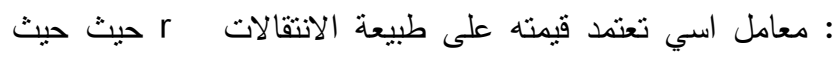

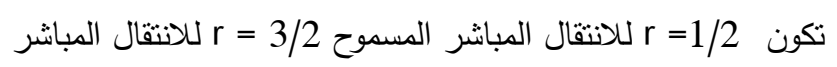
الممنوع

ع - حساب فجوة الطاقة المحظورة للانتقالات الاككترونية غبر

$$
\text { مباشرة : n }
$$

يتم حساب فجوة الطاقة المحظورة للانتقالات الالكترونية غير

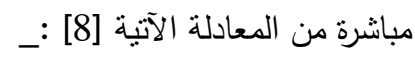

$$
\begin{aligned}
& \alpha h v=B\left(h v-\mathrm{E}_{g} \pm \mathrm{E}_{p h}\right)^{r} \\
& \text { B ثابت بعتمد على نوع المادة } \\
& \text { Eph }
\end{aligned}
$$

士 الإثارة الموجبة تعني امتصاص فونون والإثـارة السالبة تعني

$$
\text { انبعاث فونون }
$$

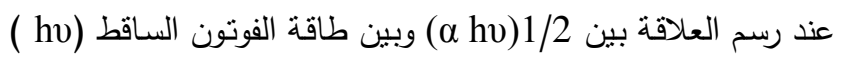

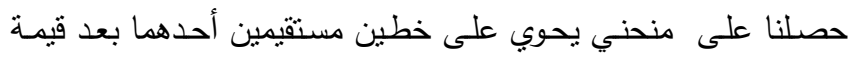

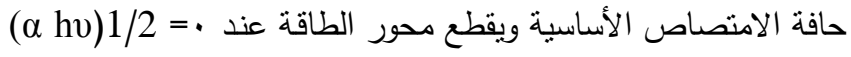

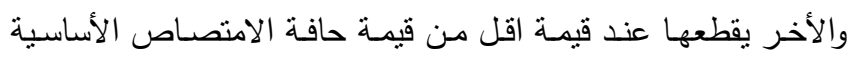
والذي يمكن من خلاله حساب طاقة الفونون المساعد ـ وبنفس الطريقة وجد ان فجوة الطاقة المحظورة للانتقالات الالكترونيـة غير المباثـرة

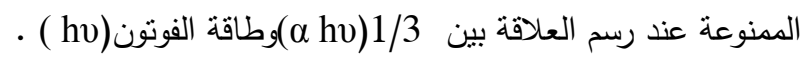

(SnO2) واطئة جدا [6]. إضافة إلى استخدامها ككواشف للأشعة فوق البنفسجية

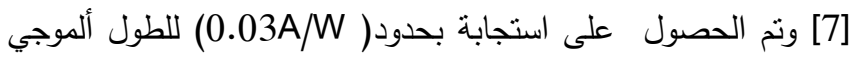

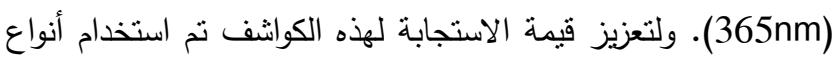

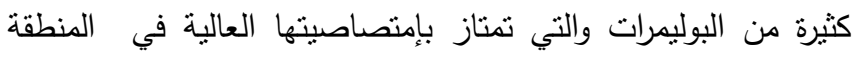
الطيفية الفوق بنفسجية فوق المنطقة الحساسة للكاشف (SnO2) ووجد إن قيمة الحساسية والاستجابة للكاثف تتحسن بصورة واضحة [8]. كذلك تم تحضير اغشية ( SnO2) الرقيقة بطريقة الطلاء بالبرم ودراسة

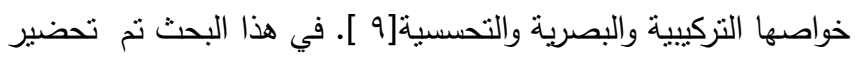
غشاء (SnO2) الرقيق بطريقة الرش الكيمبائي الحراري ومنه تم تصنيع كاثف التوصيل الضوئي للأشعة فوق البنفسجية ودراسة خصائصه

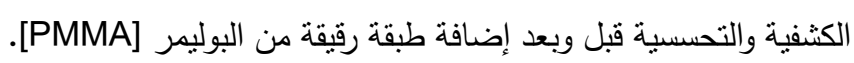

\section{الجزء النظري}

الخصائص البصرية

1-النفاذية والامتصاصية البصريه

عندما تكون طاقة الفوتون الساقط أقل من فجوة الطاقة لشبه الموصل

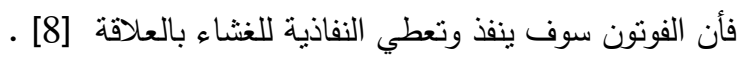
$T=(1-\operatorname{Re}) \exp (-\alpha \mathrm{t})$

حيث أن : T : النفاذية .

. Re

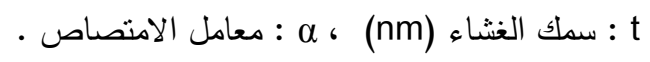

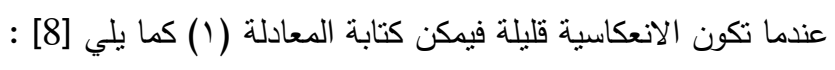

$$
T=\exp (-\alpha \mathrm{t})
$$

أما الامتصاصية فإنها نساوي [8] :

$$
A=\log 10(1 / T)
$$

$$
\begin{aligned}
& \text { حيث A :الامتصاصية } \\
& \text { يمكن حساب الانعكاسية من المعادلة الآتية } \\
& \mathrm{Re}+\mathrm{T}+\mathrm{A}=1
\end{aligned}
$$

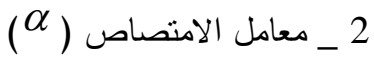

يعرف معامل الامتصاص Absorption Coefficient : بأنه الماصن 
تم قياس الخصائص البصرية للأغشية الرقيقة من خلال دراسة طيف

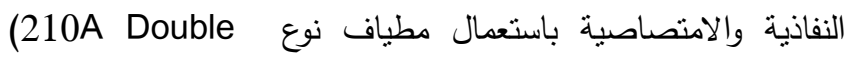
Beam Spectrophotometer U.V visible) الطول ألموجي (320-850nm) ونم استخدام جهاز حيود الاشعه السينية نوع Shimadzu (XR-Difractometer-6000) لمعرفة نوعية التركيب البلوري للأغشية الرقيقة .إما جهاز AFM فتم استخدامه

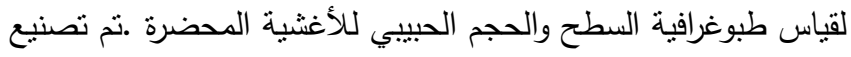
كاشف التوصيل الضوئي للأشعة الفوق بنفسجية من أغشية (SnO2) بترسيب أقطاب الألمنيوم على تلك الأغثية باستخدام طريقة التبخير

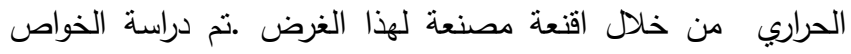

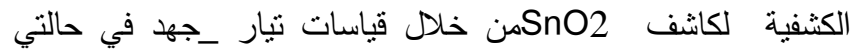

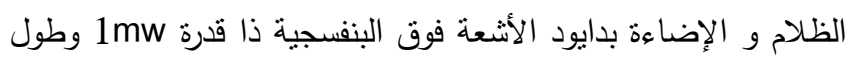
موجي 385nm ونم قياس الاستجابة الطيفية .لتحسين الاستجابة

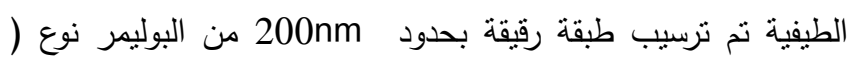

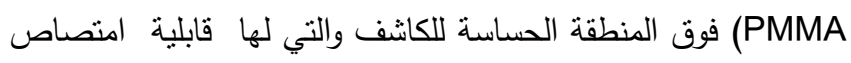
عالية للأشعة فوق البنفسجية.

\section{النتائج والمناقشية}

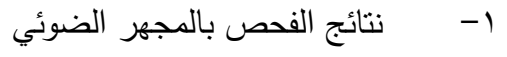

بيين الثكل (1) صور باستخدام ألمجهر الضوئي لأغشية (SnO2) الرقيقة قبل وبعد التلدين .نلاحظ في هذه الصور وجود تفرعات شجيرية تشير إلى مراحل نمو بدائية قد تحصل للغشاء .لوحظ من هذه فئه

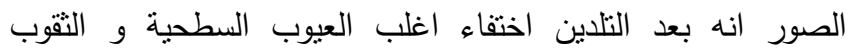
وظهور الحدود الحبييية بشكل واضح ،وهذا يشير إلى تحسن التركيب البلوري للغشاء إي تحسن في خصائص الغشاء.
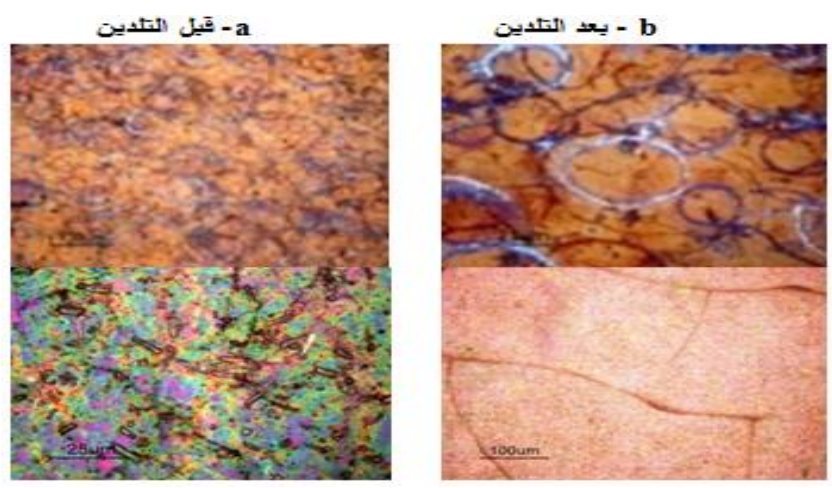

الشكل (1) صور المجهر الضوئي لغثاء SnO2 قبل وبعد التلدين عند - بعد التلدين - b

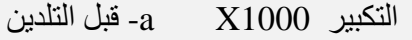

ع _ حساب الاستجابة الطيفية والربح: Spectral responsivity

and gain calculations

يمكن حساب الاستجابة الطيفية للكواشف بأستخدام المعادلة التالية [8]:

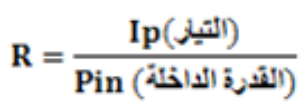

حيث التبار الضوئي ، Pin IP الضوء الساقط. كذلك فأن الربح للتيار الضوئي يمكن إن يحسب بمارئي

$$
\mathbf{G}=\frac{\mathbf{I}_{\mathbf{P}}}{\mathbf{I}}
$$

حيث ا تيار الظلام (dark current).

\section{الجزء العملي}

يتم أولا تحضير القواعد الزجاجية و تتظف بالماء المقطر وكحول

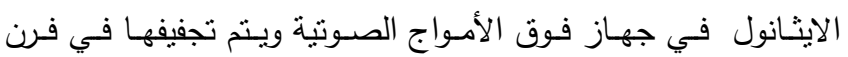

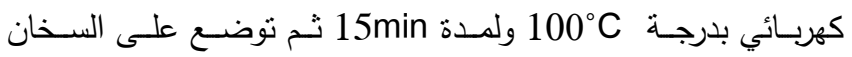

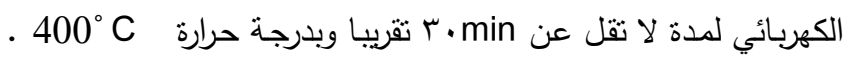

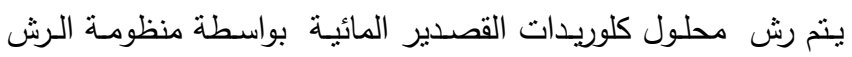

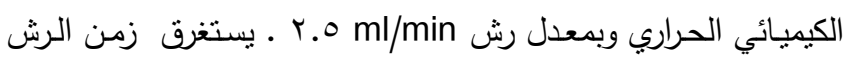

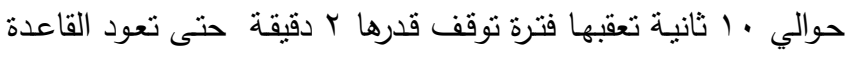

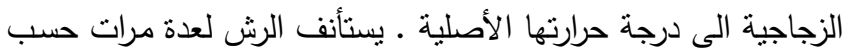

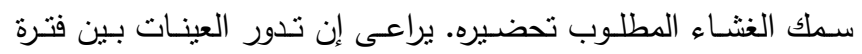

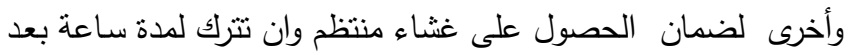

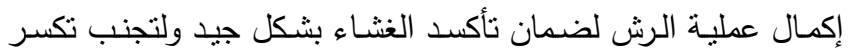

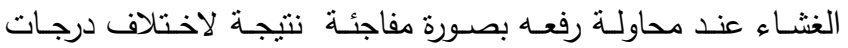

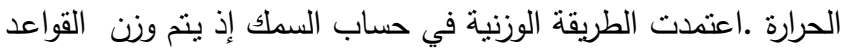

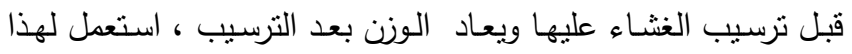

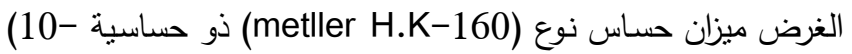
( ومن معرفة مساحة وكثافة المادة المرسبة ويمكن حساب 4 gm)

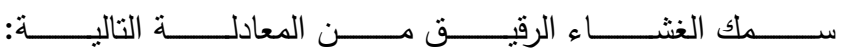
$\mathbf{t}=\frac{\Delta \mathbf{m}}{\boldsymbol{\rho} \mathbf{A}_{\mathbf{r}}}$

$$
\text { حيث إن: }
$$

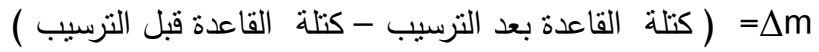
م= كثافة الغثاء المرسب Ar 
(قيل التثلين)

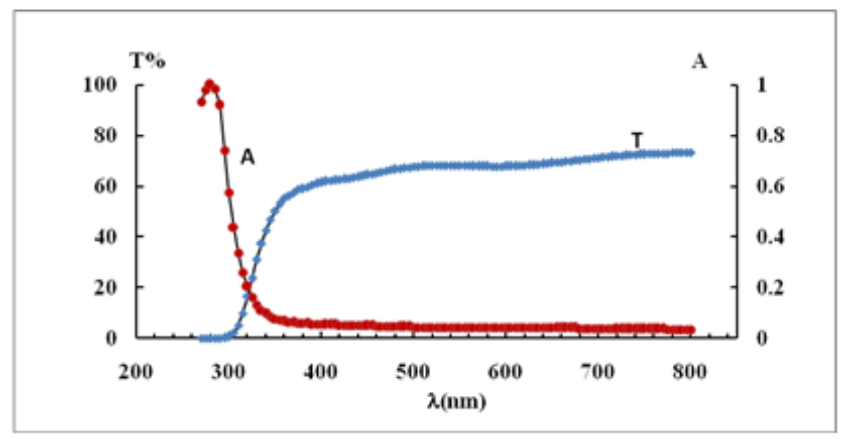

(بع التملثين)

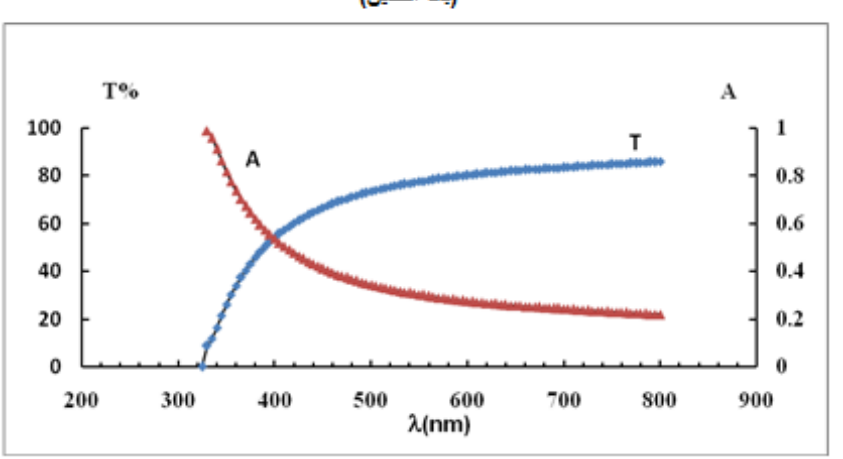

الشكل(ץ) طيف النفاذية والامتصاصية بوصفه دالة للطول الموجي عند سمك $300 \mathrm{~nm}$

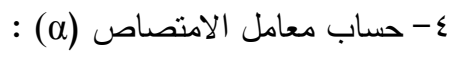

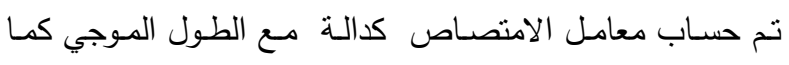

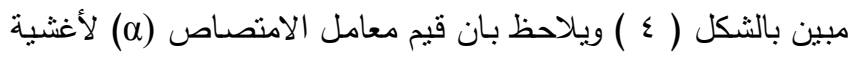
هي بحدود ( SnO2) المنحني يكون فيه تغير معامل الامتصاص بطيئا في منطقة الطاقات

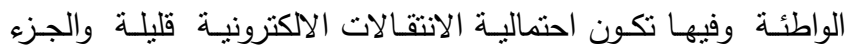

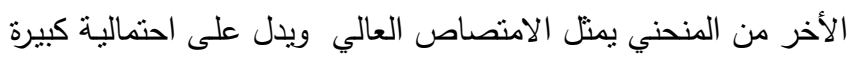
للانتقالات الالكثرونية وهذه المنطقة تمثل حافة الامنصـاص الآسية. لوحظ بـان التلدين ادى إلى تقليل قيمـة الامتصاصية (تقليل معامـل الامتصاص م ) والسبب في ذللك يعزى إلى زيادة درجة بلورية الغثاء الرقيق نتيجة لزيادة حجم الحبيبات بعد التلادين. r- بنتائج فحص الأشعة السينية:

تم التعرف على تركيب الأغشية المحضرة من خلال دراسـة الثكل( r ) الذي يبين العلاقة بين شدة الاشعة السينية الساقطة بوحدات

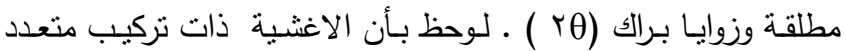
التبلور ومن النوع الرباعي وباتجاهية سـائدة (110,101,200,211) قبل وبعد التلدين .ويلاحظ من هذا الثنكل الارتفاع الواضح للقمم بعد التد التلدين ويعزى ذلك إلى تحسن التبلور وتتاقص العيوب البلورية.
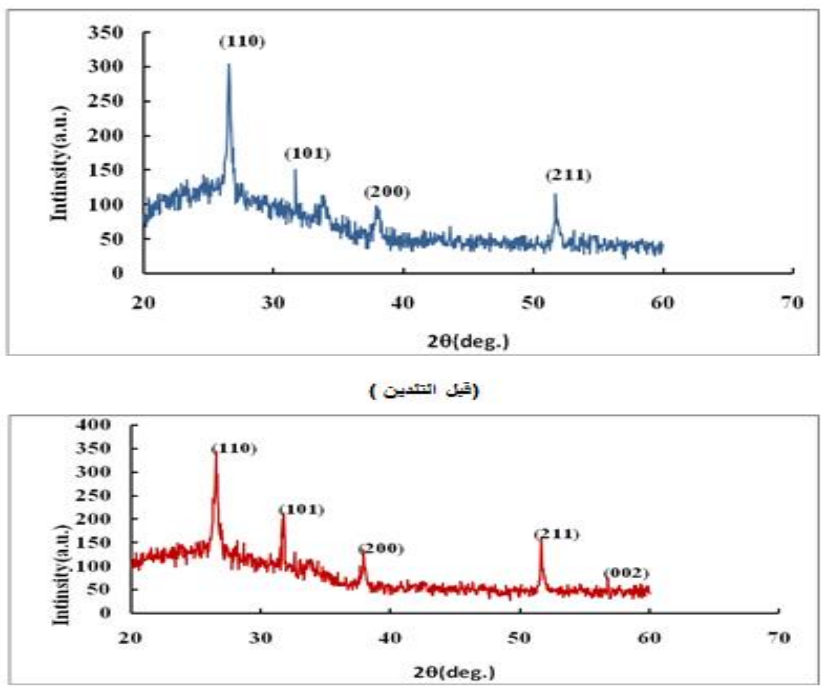

(بع التئنين)

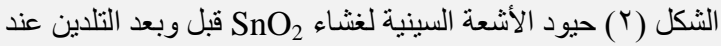
سمك $300 \mathrm{~nm}$

طيف النفاذيـة والامتصاصية : أجريت قياسـات طيف الامتصاصية

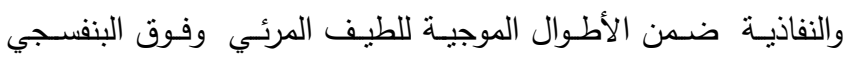

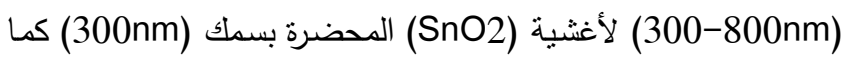
هو مبين بالثكل (3) حيث يلاحظ إن الامتصاصية تكون ذات قيمة

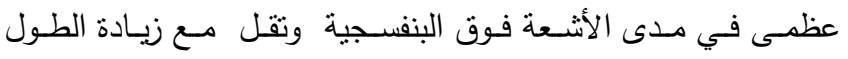

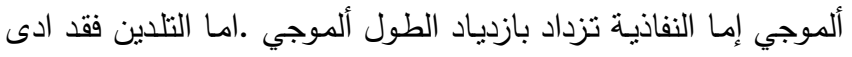

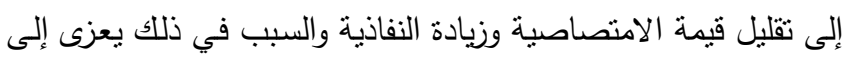

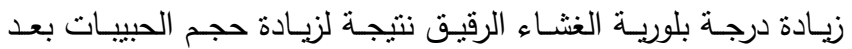




$$
\text { 7- القياسات الكثنية : }
$$

مواصفات البوليمر المستخدم :هو من النوع بولي مثيل ميثا

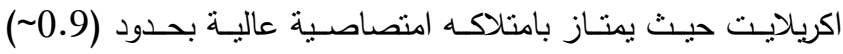

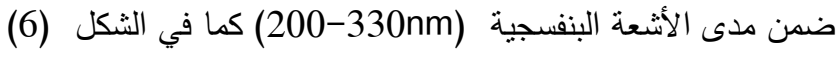

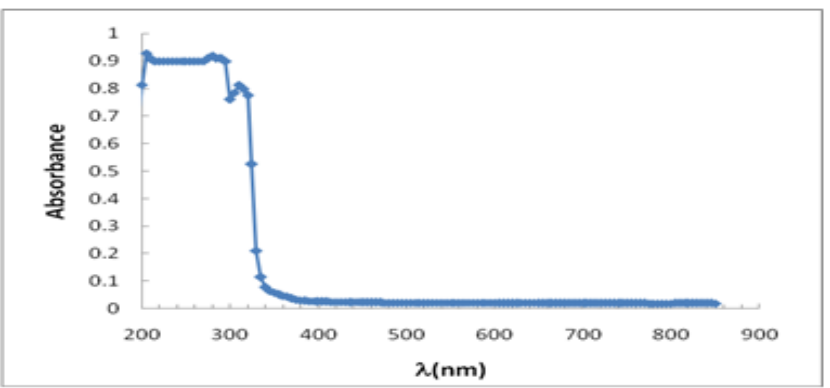

$$
\text { الثكل (ך) طيف الامتصاصية للبوليم ( PMMA) }
$$

1-1 : خصائص التيار فولتية :

تم حساب خصائص التيار -فولنية للكاشف المصنع عند تعرض

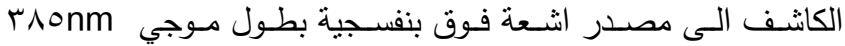

وطاقة حوالي mW I يلاحظ من الثكل (7) بان تبار الظلام

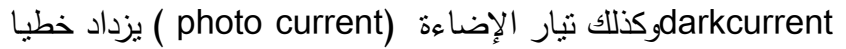
بزيادة الفولتية المسلطة وهذا يشير إلى الطبيعة الاومية للكاثف ـ ومن

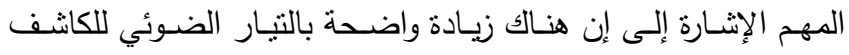
(SnO2)

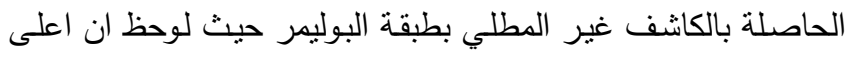

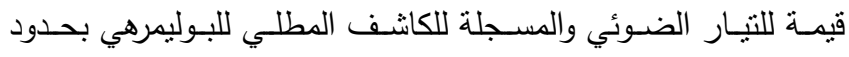
.$(1620 \mu \mathrm{A})$

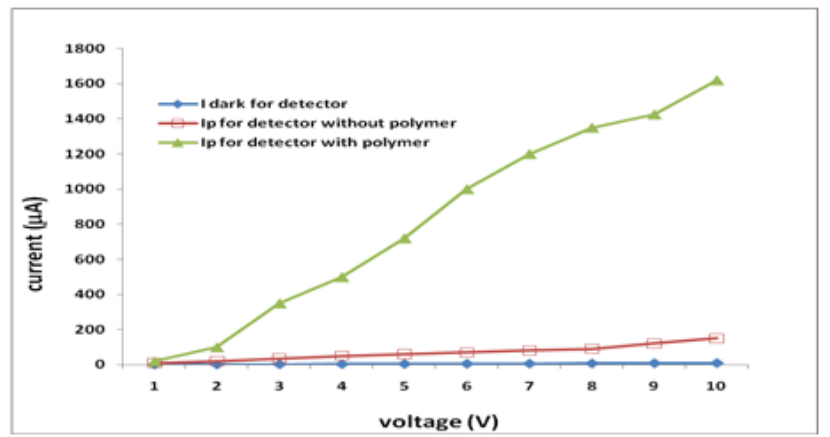

الثكل (VnO2 العلاقة بين التيار و الفولتية لكاشف
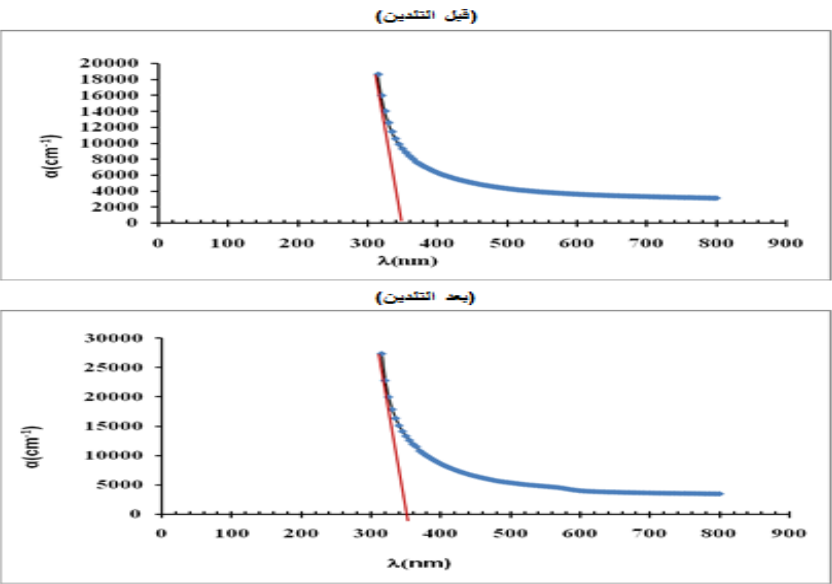

الثكل(4)معامل الامتصاص بوصفه دالة للطول ألموجي لغشاء

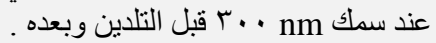

0- حساب فجوة الطاقة :

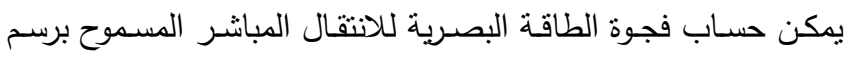
العلاقة الخطية بين rhv( ) وبين طاقة الفونون الساقط (hv) كما هو

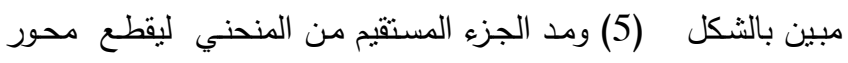

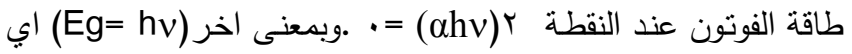
ان نقطـة القطـع تمثنل قيمـة فجـوة الطاقـة المنوعـة (Eg) للانتقـال

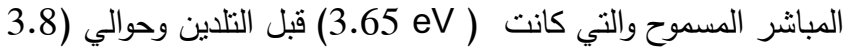
(eV بعد التلدين وذلك يعزى الى ان التلدين ادى إلى زيـادة تبلـور الغشاء وتقليل العيوب البلورية وبالتالي تقليل المستويات الموضعية بين حزمني التكافؤ والتوصيل مما يؤدي إلى زيادة فجوة الطاقة الممنوعة.

(3) (التثين )
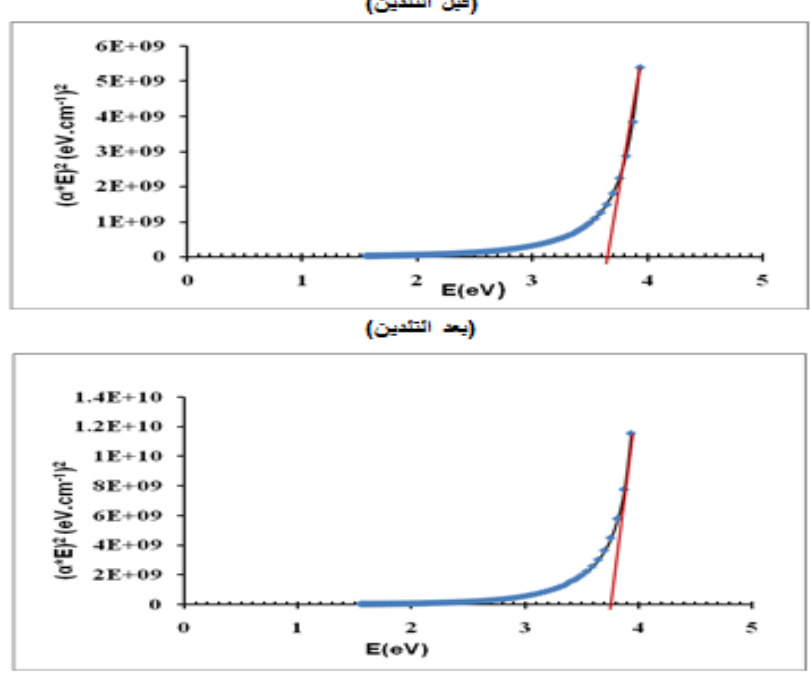

الثكل (5 ) فجوة الطاقة للانتقال المباشر المسموح لغشاء SnO2 عند سمك 300nm قبل التلدين وبعده. 


\section{References}

[1] T.W.Kim, D.V.Lee, D.C.Choo and Y.S.Yoon, "Structural, electrical and optical properties of $\mathrm{SnO} 2$ nanocrystalline thin films grown on $\mathrm{InSb}$ substrate"J.Appl.Phys.Vol90, 1(2001)

[2] M.Batzill and U.Diebold, "The surface and materials science of tin oide" Progress in surface science 79, p 147 (2005)

[3] H.S.Rrandhawa, M.D.Mathews and R.F.Bunshah " $\mathrm{SnO}_{2}$ films prepared by activated reactive evaporation" Thin Solid Film, 83, 267 (1981)

[4] M.M.Bagheri and M.shokooh ," Investigation on the physical properties of $\mathrm{SnO} 2-\mathrm{ZnO}$ Transparent conducting binary system depositedby spry pyrolysis technique "THIN FILMS ,441,P238 (2003)

[5] K.L.Chopra and I.Kaur," Thin film device applications"PLENUM PRESS, NEWYORK (1983)

[6]C.Wang, L.Yin and L.Zhang,"metal oxide gas sensors: sensitivity and influencing factor " Sensors, V.10.N.20 (2010).

[7] L .Lous,S.S Mao and L.Lin ," Fabrication and characterization of ZNO Nanowires based UV photodiodes "SENSORS AND ACTUTORS, A-127 P-201(2006)

[8] C.Shi,M.Park ,Y.Deng,and .Z.L.Warg,"Giant enhancement in UV response of $\mathrm{ZnO}$ Nanobelts by polymer surface-functionalization" .J.AM.CHEM.SOC,129,P-12096(2007)

[9] S.Majumder,"Synthesis and characterization of SnO2films obtained by a wet chemical process"Material Science, V.27, N.1 (2009).
T- Y T : الاستجابة الطيفية :

تم حساب الاستجابة الطيفية للكاثف المصنع باستخدام المعادلة (9)

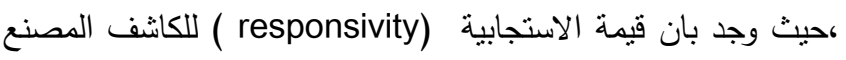

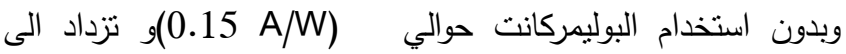
nm عند طلاء الكاشف بطبقة رقيقة من البوليمربسك (1.62 A/W)

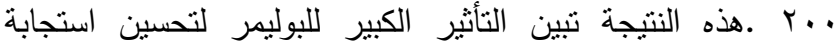
الكاشف.ويمكن تعليل ذلك إلى إن البوليمر أدى إلى استحداث SnO2 Sمتويات طاقية جديدة والتي تقع ضمن فجوة الطاقة لمادة كمستويات موضعية تزيد من احتمالية استثارة وانتقال الالكترونات إلى لى حزمة التوصيل عند تعريض الكاثف إلى الأشعة. وبالتالي زيادة الاستجابية للكاثف نتيجة لزيادة التيار الضوئي الناتج. نم حساب قيمة الربح للكاشف المصنع باستخدام المعادلة (• (1) وكانت قيمتها (. (1) بالنسبة للكاشف غير المطلي بالبوليمر و (.1 • (1) للكاثن المطلي

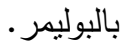

\section{الاستتناجات}

1- تبين من نتائج فحوصات الاثعة السينية ان أغشية SnO2 الرقيقة

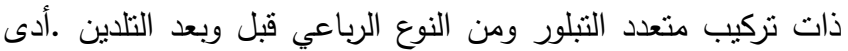
التلدين إلى زيادة النفاذية وقلة الامتصاصية وبالنالي قلة معامل

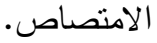
r- زيادة قيمة فجوة الطاقة الممنوعة (Eg) للانتقال المباشر المسموح

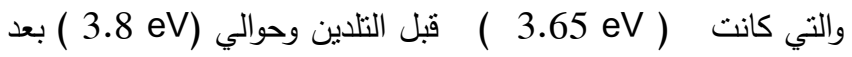
التلدين .ادى التلدين الى زيادة تبلور الغشاء وتقليل العيوب البلورية

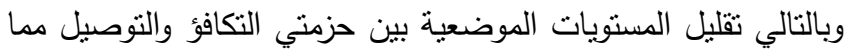
يؤدي الى زيادة فجوة الطاقة الممنوعة. r- ـلاء كاثف الأشعة فوق البنفجية بطقة رقيقة من البوليمر نو

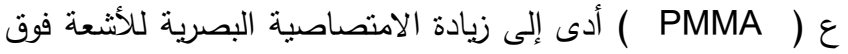
البنفسجية وبالثالي تحسين قيمة الاستجابية الطيفية والربح الكلي للكاشف المصنع • إن الربح الكلي للكاثف المصنع يصل إلى قيمة عظمى بعد طلاء الكاثف بالبوليمر إما أعظم استجابة طيفية بحدود 